\title{
Handling uncertainties in production network design
}

\author{
Günther Schuh ${ }^{1}$, Jan-Philipp Prote ${ }^{1}$, Andreas Gützlaff ${ }^{1}$, Sebastian Henk ${ }^{1}$ \\ ${ }^{1}$ Laboratory for Machine Tools and Production Engineering (WZL) of \\ RWTH Aachen University, 52074 Aachen, Germany \\ \{g.schuh,j.prote,a.guetzlaff,s.henk\} \\ @wzl.rwth-aachen.de
}

\begin{abstract}
Decision making in production network design is complex due to a large number of influencing factors, options and uncertainties. Furthermore, the agility in production networks and therefore the decision demand increases while made decisions are often hard to revise. Hence, a fast yet holistic decision-making process is key for sustainable production network development. While many existing approaches target the overall network optimization, few of them include a systematic approach to cover uncertainty and barely any approaches cover the uncertainty of information and models used for the decision-making. In praxis, these approaches result in unsystematic and time-consuming decision-making processes. This paper presents an approach to take uncertainty systematically into consideration and splits it into internal uncertainty, which can be reduced by the decision maker, and external uncertainty, which has to be considered in the sensitivity analysis. The method was applied for the site selection of an automotive supplier.
\end{abstract}

Keywords: Production networks, decision process, uncertainty

\section{Introduction and Motivation}

As globalization progresses, companies are trying to grow by internationalizing their business activities and, at the same time, become less dependent on local economic fluctuations. This development is reflected in an increasing number of production locations for manufacturing companies [1]. Manufacturing companies are also increasing their market share across an increasing range of products. At the same time, product life cycles are shortening. An increasing number of variants and a decreasing number of identical parts are created in production [2].

Due to the global distribution of added value and the increasing product complexity, production networks today are among the most complex and dynamic man-made systems, which often results in historically grown structures [3]. At the same time, location decisions are difficult to revise, which is why decisions have to be made carefully [4].

Existing approaches for production network design generate a large operational modelling effort in order to tackle the complexity and impact of decisions in the network (see 2.1). However, the approaches often lack a holistic view of uncertainty. Decisions are therefore often prolonged by unsystematic additional analyses [5]. The aim 
of this paper is to present an approach for assessing uncertainty in the planning process of production networks. This enables a focus on the most important factors of the decision and the planning time can be significantly shortened by the absence of unnecessary iterations.

\section{State of the Art}

\subsection{Existing Approaches for Production Network Design}

Current approaches for production network design can be distinguished between models which describe how to proceed in production network design, optimization and simulation models and - as a new trend within the last years - big data driven analysis approaches:

Procedure models describe consecutive steps to create and evaluate production network scenarios. LANZA et al. give an overview of recent approaches [6].

CHRISTODOULOU et al. for example describe a multi-stage approach for network design that proposes a complexity reduction by defining simplifying assumptions and splitting the network into subnetworks for each product group. The approach emphasizes the importance of creating reliable production cost models and finding a realistic demand forecast, but does not further detail how to achieve this. [7]

STOFFEL developed a V-Model to design large production networks that focuses on a strategy based reconfiguration of the whole network. It structures the information need and the level of granularity but also does not cover uncertainty in the decision making process systematically. [8]

Optimization models are the most commonly described method to design production networks [6]. A detailed overview of optimization approaches is given by CHENG et al. [9] and OlHAGER et al. [10]. LANZA and MOSER for example provide a detailed approach that evaluates the impact of changing influencing factors and includes objectives such as delivery time, quality, flexibility and coordination effort [11]. Even though they give an optimal solution from a mathematical point of view, optimization models do not recognize wrong input data and have to quantify and rank qualitative network design objectives.

Simulation models support the decision making process by providing information about complex interactions, e.g. about the robustness of a network scenario regarding different production volumes as demonstrated by PUTNIK et al. [12]. This models can be helpful to decrease uncertainty, but are not suitable to systematically create network alternatives.

Big data analyses like presented by GöLZER et al. [13] try to provide decision support systems based on the analysis of large data sets. An overview of recent approaches is given by TIWARI et al. [14]. These approaches might help to gather information faster, but neither can they provide information about the reliability of the data sets nor can they consider qualitative aspects of production network design. 


\subsection{Handling Uncertainties}

In general, proper decision making requires the consideration of environmental states [15]. These conditions are often not known with certainty. Thus, uncertainty represents any deviation from the ideal of complete knowledge [16]. There are various ways to account for uncertainties in decision making. Those can be classified between the two extremes of totally neglecting uncertainties and solving complex stochastic problems. The goal is to find a good compromise between planning effort and result [17].

To identify and prioritize critical uncertainties, WALKER et al. distinguish three dimensions of uncertainty to enable an adequate consideration in the decision-making process [16]: Nature of uncertainty, Level of uncertainty and Location of uncertainty.

The nature of uncertainty focuses on the origin and describes whether the uncertainty is based on a lack of knowledge (epistemic uncertainty) or on inherent variability (variability uncertainty). While the former can be reduced by further research and empirical efforts, latter remains due to natural randomness as well as behavioral and societal variability [16]. The level of uncertainty describes how the uncertainty is ranked within a seven-level spectrum between deterministic knowledge and total ignorance. Depending on the level of uncertainty, a statistical or scenario consideration is required as shown in Fig. 1 [18].

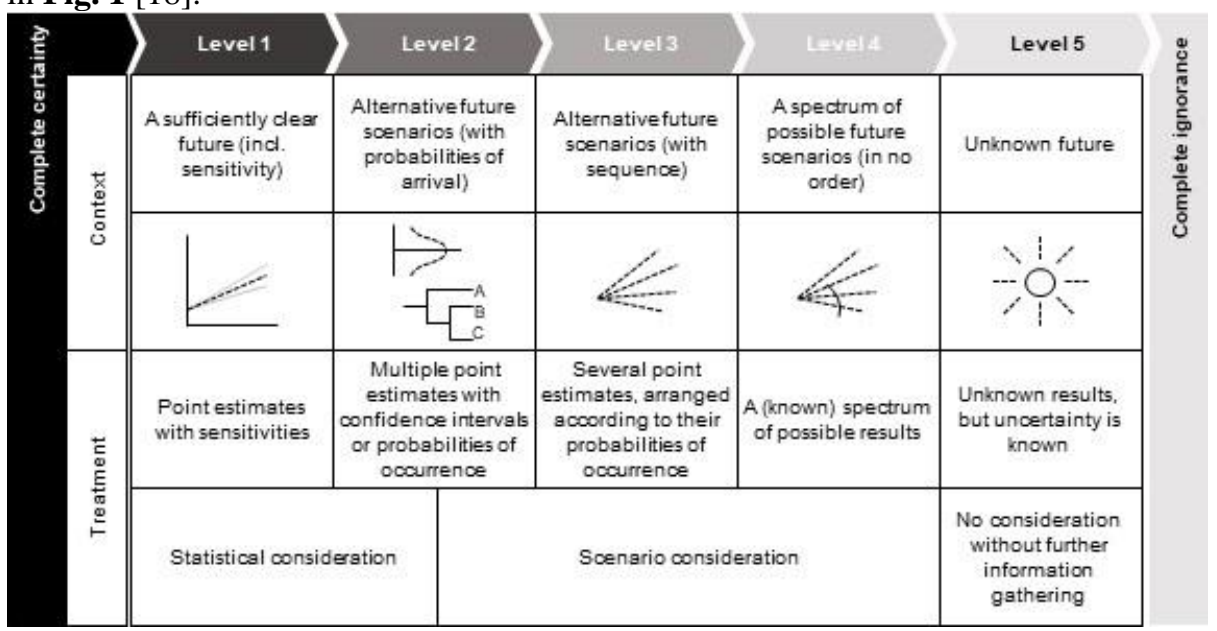

Fig. 1. Levels of uncertainty by WALKER et al. [18]

The location of uncertainty characterizes where the uncertainty manifests itself within an existing or designed model complex. There are four different generic locations of uncertainty [19]: Context uncertainty, Data uncertainty, Model uncertainty and Phenomenological uncertainty. While context uncertainty covers the endogenous and exogenous uncertainties which are caused by surrounding influences [20], data uncertainty describes uncertainties due to data incompleteness, data inaccuracy and variation in the input data [21]. In addition, the inaccuracies of a simplifying model in compari- 
son to reality lead to model uncertainties containing conceptual, mathematical and computational model uncertainties. Phenomenological uncertainty affects the consequences of a decision [19].

\section{Approach}

In order to systematically consider uncertainty in production network design, the presented approach follows the decision making process by SPETZLER [5] and distinguishes the phases of initialization, information gathering, alternatives creation, evaluation and decision making (Fig. 2). Based on this steps, uncertainty factors are identified and prioritized to focus the analysis effort and the evaluation scope.

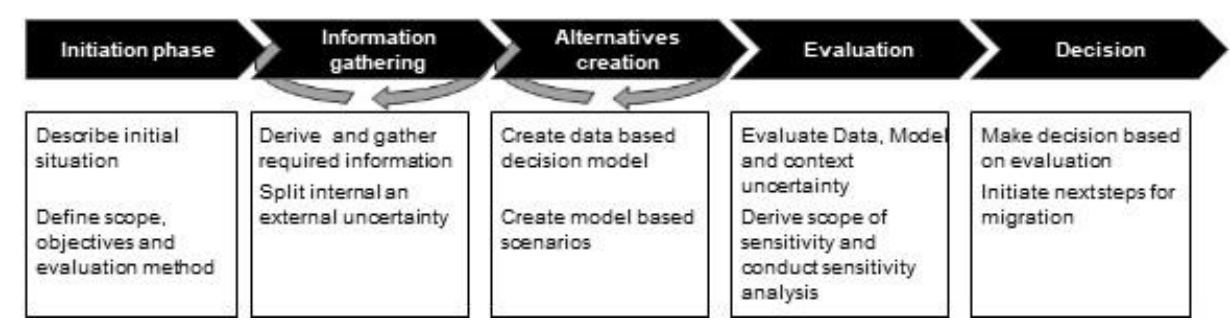

Fig. 2. Adaptation of the generic decision making process by SPETZLER [5] for production network design

\subsection{Initiation Phase}

The focus within the initiation phase is to transfer the generous thoughts on an upcoming need for action into a structured decision problem. By determining the general scope and the focused objectives, the following selection of the evaluation method is enabled. A rough cost structure analysis helps to identify the cost critical aspects of the decision making, e.g. the importance of transport costs or wages as part of the total landed cost. The literature contains a wide variety of evaluation methods (e.g. see SCHUH et al. [22]), which should be selected depending on the desired detail degree of the consideration and the importance regarding the cost structure. Based on the selected evaluation methods and the decision scope, the information need to apply the selected methods can be derived.

\subsection{Modelling Phase}

The modelling phase includes the steps of information gathering and alternatives creation. First, the elements and influencing factors of the evaluation method determined in the initialization phase are divided into internal and external factors on the basis of the uncertainty evaluation by WALKER et al. (Fig. 3). Internal uncertainty describes the uncertainties associated with the considerations within the decision-making process, which primarily depend on the level of detail selected. External uncertainties arise from external driven situations and developments. These are unknown to the decision maker and can only be anticipated to a limited extent by obtaining information. 


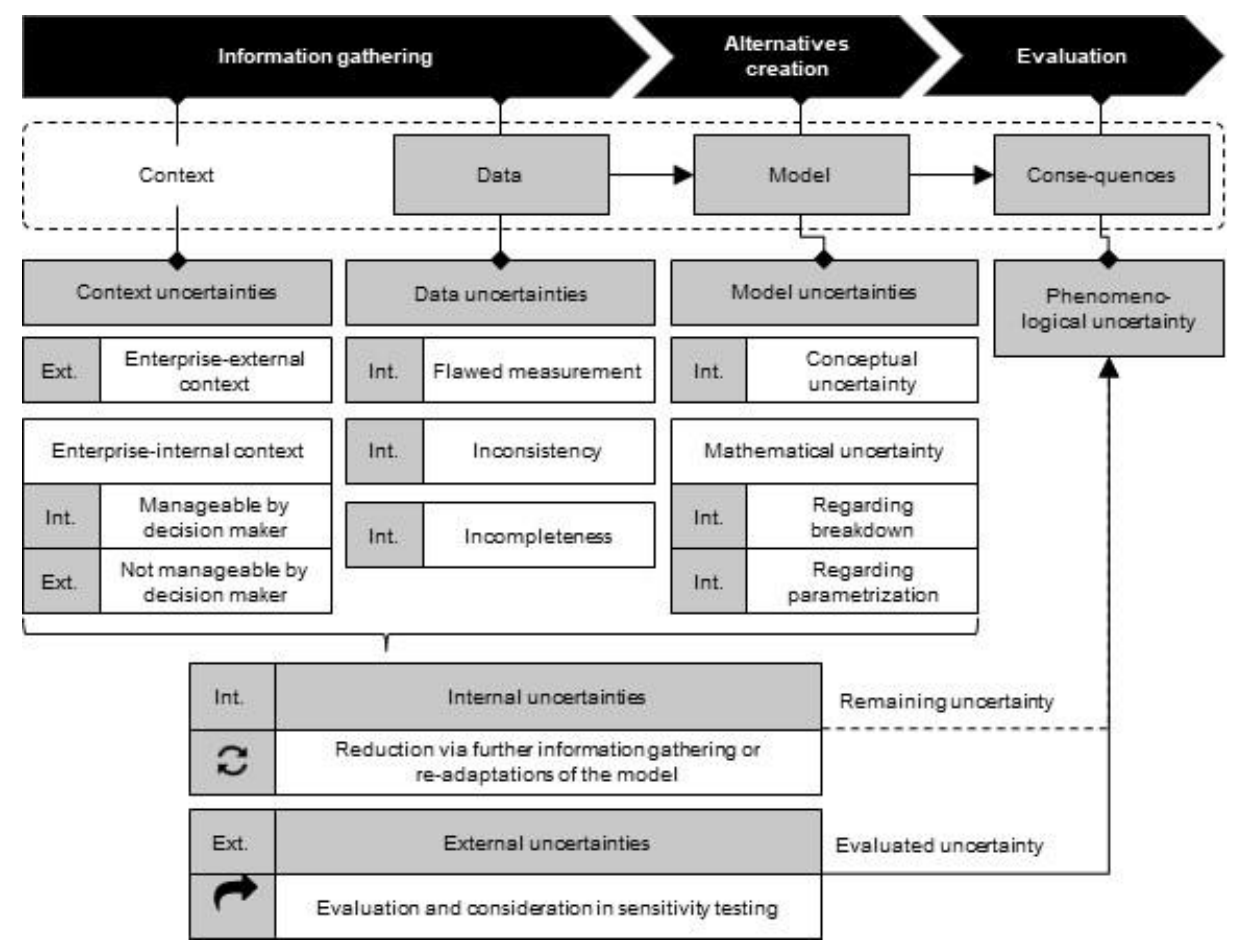

Fig. 3. Differentiation of uncertainty forms for further consideration

For the information gathering, a distinction is made between context and data. Context includes all internal and external developments that affect the decision-making process. External to the company is for example the general market development, internal the planned introduction of new products. However, a distinction must be made here as to whether this is a factor that the decision-maker can influence or not. For external context uncertainty, only an assessment can be made. Complete certainty is not possible. Historical developments can be extrapolated and supplemented by expert assessments in order to limit development opportunities. The assessment of the level of uncertainty is based on the experience of the decision maker and the importance of the influencing factor in relation to the overall decision. Depending on the assessment of the uncertainty, a sensitivity analysis of the scenarios is conducted or new scenarios are created in order to quantify various development possibilities.

Data uncertainty is broken down into flawed measurement, inconsistency and incompleteness. In contrast to external uncertainty, this raises the question of the extent to which uncertainty can be reduced by additional survey effort. This is usually possible by including additional data sources, new or additional data collection or expert surveys. The new data collection can be conducted iteratively until a sufficiently valid data basis for modelling has been created. While the final assessment of the sufficient validity depends on the risk affinity of the decision maker, it is necessary to consider the remaining uncertainty in the evaluation phase in order to achieve a high decision quality. It is important to achieve the required accuracy in this step before moving on to 
modelling, as otherwise the uncertainty of the data is transferred to the model uncertainty and the model cannot be calibrated.

For alternatives creation, a calibration of the as-is model with the existing network is necessary to determine the model uncertainty. The error deviation can be used for an iterative calibration of the model. The remaining uncertainty has to be mapped by sensitivity analyses after alternative scenarios have been created in the next step. If the uncertianty is very high, additional scenarios should be considered (Fig. 2). For the scenario generation, existing methods such as the previously introduced approach "Making the right things in the places" can be used [7].

\subsection{Evaluation and decision making}

In the final step, the alternatives are converted into evaluation scenarios with sensitivities according to scenario techniques like the one developed by GAUSEMEIER et al. [23]. All relevant influences are taken into account with their inherent uncertainty. The range of sensitivity per influencing factor depends on the uncertainty in the previous evaluation process. The final decision is based on the action alternatives and their sensitivities regarding the focused objectives. A holistic approach to scenario assessment based on Economic Value Added is presented by SCHUH et al., for example [22]. There can be two different results in the decision: Either there is a dominant scenario, or there are different scenarios that cannot be finally weighed against each other due to their sensitivity. In the latter case, it should be examined whether further sources are available to reduce the internal uncertainty. Otherwise, it is advisable to choose the scenario preferred from a strategic point of view.

\section{Application}

The expansion or opening of a press shop was planned for the production of body parts for a high-volume model of an OEM. During the initialization phase, the scope of consideration could be narrowed down to one existing or two potentially new plants: A low transport and a low cost site. As the sales price was fixed, the valuation was based on the cumulated cash out over the product lifetime of 14 years. A static cost model on an annual basis was chosen for modelling purposes and iteratively specified to reduce data and model uncertainty. A rough cost estimation in the initiation phase shows that transports and wages have a higher impact on the costs than the investment in machines and buildings. The information gathering therefore focused on those two factors, while investments and fixed costs were estimated with good accuracy on the basis of previous projects. While wage costs could be determined with good accuracy, transport costs are difficult to estimate, especially due to the long planning period and external factors. Therefore, a broad sensitivity corridor hat to be taken into account. The model results in a dominant scenario for the existing location, but due to the sensitivity of transport costs it is not absolutely dominant (Fig. 4). The management chose the dominant scenario due to lower investments and therefor lower risks of sunk cost effects. The entire decision making process took two weeks. 


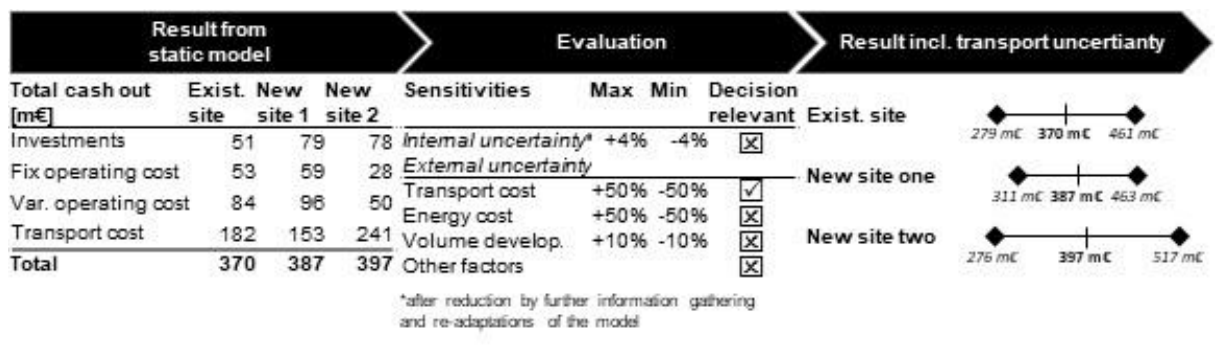

Fig. 4. Results of the method application

\section{Conclusion and Future Research}

A focused decision-making process can be derived from a preliminary analysis of the decision problem and an uncertainty-oriented procurement of information. By recording and evaluating the uncertainty in information acquisition and modelling, unnecessary detailing loops can be avoided by mapping the uncertainty in the sensitivity analysis or through additional scenarios. Detailed information is only obtained for the most relevant factors. Thus, even investment-intensive, far-reaching decisions can be made in a short time. The assessment of uncertainty, however, continues to depend on the experience and knowledge of those involved in the process and cannot be completely quantified. Further research is therefore needed to derive concrete specifications for the classification of uncertainty in relation to sensitivities and scenario alternatives.

\section{Acknowledgment}

The authors would like to thank the German Research Foundation DFG for the kind support within the Cluster of Excellence "Internet of Production" - Project-ID: 390621612 .

\section{References}

[1] Schuh, G., Reuter, C., Prote, J.-P., Stöwer, M., Witthohn, C., Fränken, B., 2016. Konsortial-Benchmarking: Gestaltung von globalen Produktionsnetzwerken.

[2] Roland Berger Strategy Consultants, 2012. Mastering product complexity.

[3] Váncza, J., 2016. Production Networks, in: Produ, T.I.A.f., Laperrière, L., Reinhart, G. (Eds.), CIRP Encyclopedia of Production Engineering. Springer, pp. 1-8.

[4] Krystek, U., 1997. Internationalisierung: Eine Herausforderung für die Unternehmensführung, XXI, $617 \mathrm{~S}$.

[5] Spetzler, C.S., 2016. Decision quality: Value creation from better business decisions. John Wiley \& Sons Inc, Hoboken, New Jersey, 1 p. 
[6] Lanza, G., Ferdows, K., Kara, S., Mourtzis, D., Schuh, G., Váncza, J., Wang, L., Wiendahl, H.-P., 2019. Global Production Networks: Design and Operation, in: CIRP Annals 68 (2).

[7] Christodoulou, P., Fleet, D., Hanson, P., Phaal, R., Probert, D., Shi, Y., 2007. Making the right things in the right places: A structured approach to developing and exploiting 'manufacturing footprint' strategy.

[8] Stoffel, M., 2016. V-Modell zur Auslegung großer Produktionsnetzwerke, Apprimus Verlag, Aachen.

[9] Cheng, Y., Farooq, S., Johansen, J., 2015. International manufacturing network: Past, present, and future. Int Jrnl of Op \& Prod Mnagemnt 35 (3), 392-429.

[10] Olhager, J., Pashaei, S., Sternberg, H., 2015. Design of global production and distribution networks. Int Jnl Phys Dist \& Log Manage 45 (1/2), 138-158.

[11] Lanza, G., Moser, R., 2014. Multi-objective optimization of global manufacturing networks taking into account multi-dimensional uncertainty. CIRP Annals 63 (1), 397400.

[12] Putnik, G.D., Škulj, G., Vrabič, R., Varela, L., Butala, P., 2015. Simulation study of large production network robustness in uncertain environment. CIRP Annals 64 (1), 439-442.

[13] Gölzer, P., Simon, L., Cato, P., Amberg, M., 2015. Designing Global Manufacturing Networks Using Big Data. Procedia CIRP 33, 191-196.

[14] Tiwari, S., Wee, H.M., Daryanto, Y., 2018. Big data analytics in supply chain management between 2010 and 2016: Insights to industries. Computers \& Industrial Engineering 115, 319-330.

[15] Laux, H., Gillenkirch, R.M., Schenk-Mathes, H.Y., 2014. Entscheidungstheorie, Springer Gabler, Berlin.

[16] Walker, W.E., Harremoës, P., Rotmans, J., van der Sluijs, J.P., van Asselt, M.B.A., Janssen, P., Krayer von Krauss, M.P., 2003. Defining Uncertainty: A Conceptual Basis for Uncertainty Management in Model-Based Decision Support. Integrated Assessment 4 (1), 5-17.

[17] Klein, R., Scholl, A., 2012. Planung und Entscheidung: Konzepte, Modelle und Methoden einer modernen betriebswirtschaftlichen Entscheidungsanalyse, München.

[18] Walker, W.E., Lempert, R.J., Kwakkel, J.H., 2013. Deep Uncertainty, in: Encyclopedia of Operations Research and Management Science. Springer, Boston, pp. 395402.

[19] Kreye, M.E., Goh, Y.M., Newnes, L.B., 2011. Manifestation of uncertainty - A classification, in: Proceedings of the 18th International Conference on Engineering Design (ICED 11), pp. 96-107.

[20] Weck, O. de, Eckert, C., Clarkson, J., 2007. A classification of uncertainty for early product and system design, in: Proceedings of the 16th International Conference on Engineering Design (ICED 07), Paris, pp. 159-171.

[21] Huijbregts, M.A.J., Norris, G., Bretz, R., Ciroth, A., Maurice, B., Bahr, B. von, Weidema, B., Beaufort, A.S.H. de, 2001. Framework for modelling data uncertainty in life cycle inventories. Int J LCA 6 (3), 127-132.

[22] Gausemeier, J., Fink, A., Schlake, O., 1996. Szenario-Management: Planen und Führen mit Szenarien, Hanser, München. 